Hypothesize Once, Plan Twice

Erin M. Buchanan, Harrisburg University of Science and Technology

K. D. Valentine, Massachusetts General Hospital, Harvard Medical School

Jeffrey Pavlacic, University of Mississippi

Corresponding author:

Erin M. Buchanan, Professor

Harrisburg University of Science and Technology

ebuchanan@harrisburgu.edu 


\begin{abstract}
Preregistration has been touted as the solution to the "reproducibility crisis" (Wagenmakers, Wetzels, Borsboom, van der Maas, \& Kievit, 2012) and part of the "renaissance" in the social sciences (Nelson, Simmons, \& Simonsohn, 2018). For preregistration, researchers describe the study plan before the data is collected or the analyses have been examined. The focus of preregistration has been on solving prediction versus postdiction (i.e., hypothesizing after results are known or HARKING; Kerr, 1998) and negating flexibility in statistical analyses (i.e., p-hacking and questionable research practices; Simmons, Nelson, \& Simonsohn, 2011). We suggest that instead of solely focusing on preregistration, researchers should concentrate on adequate plans for research detailing a strong relationship between hypotheses, methods, data, and analyses. While preregistration encourages this process, more effort should focus on the research blueprint to engage in meaningful and, potentially, reproducible science.
\end{abstract}




\section{Hypothesize Once, Plan Twice}

Preregistration consists of the many components that contribute to successful psychological science (2018), simply described as detailing the study before the data are collected or analyzed. However, current shifts in scientific norms create difficulty for successful preregistration. For example, modern psychological science is largely influenced by a "publish or perish" mentality (Leis-Newman, 2011), which motivates problematic research practices (e.g., p-hacking; Gonzalez \& Cunningham, 2015; Simmons et al., 2011). While preregistration is gaining increased attention (Nosek \& Lindsay, 2018), there is substantial heterogeneity regarding the implementation of successful preregistration practices. Nosek, Ebersole, DeHaven, and Mellor (2018) detail the challenges to preregistration adoption, such as many experiments, exploratory research, and large datasets. For example, pre-existing data presents a challenge in understanding how to register a study on these data since preregistration often occurs before data collection. Preregistration templates offer a relatively easy way to meet scientific demands and curb challenges facing preregistration. Van den Akker et al. (2019) and Mertens and Krypotos (2019) have proposed templates for secondary data analysis within the context of preregistration to solve the challenges listed in Nosek et al. (2018).

Practically, what does preregistration consist of? The most parsimonious of preregistration templates includes a narrative summary with the following instructions: "Summary (optional): Provide a narrative summary of what is contained in this registration, or how it differs from prior registrations." (see https://osf.io/haadc/). The Aspredicted.org (https://aspredicted.org/) form consists of the following eight questions: (1) has the data has been collected (2) what are the hypotheses, (3) what are the dependent variables, (4) what conditions are participants assigned to, (5) what analyses will be conducted, (6) what secondary analyses are planned, (7) what is the sample size, and (8) what other considerations need to be 
made. Each question is optional. Two of the more detailed preregistrations include the Open Science Framework (OSF) Preregistration Template (https://osf.io/prereg/) and the van't Veer and Giner-Sorolla (2016) Preregistration in Social Psychology (PRSP) template. Both the OSF and the PRSP templates offer specific guidelines for hypotheses, methods, and data-analytic plans. Further, these templates address a crucial problem in many research studies, that being the lack of clarity between different components of a study. As an example, the PRSP template includes sections for describing statistical analyses used to test the prediction made in the hypotheses. Separate space exists to detail the variables and their calculation, explain covariate rationale, outline independent and dependent variables, and untangle other considerations relevant to null hypothesis testing. Ultimately, The OSF and PRSP templates offer a foundational solution to the shift in psychological science that is focused on mapping the research process to promote transparent and reproducible science.

Imagine being hired to assist in statistical analysis for a research project. How might the conversation transpire with the principal investigator? Normal questions likely fall into two categories: (1) what did you do? (2) what do you want to know? At face value, these questions appear relatively straightforward. However, complications arise when their connection is elucidated. For example, how does the data represent what you did? How were your foundational theoretical concepts measured and operationalized? What column is that in the data? Statistical analysts have difficulty aiding scientists in mapping relationships between research design, measurement, and hypotheses, among other components of research studies, given that these analysts may not be as well-versed in a given content area. This scenario is unfortunate, considering that statistical analysis is a contributor of non-replication through researcher degrees of freedom (Simmons et al., 2011). Further, the lack of structure from design and measurement to hypothesis and analysis is equally problematic, as it forces 
researchers to stretch hypotheses to fit collected data, rather than collecting the appropriate data for each hypothesis. Also exacerbating the issue, statistical analysis have "forking paths" (Gelman \& Loken, 2013) that include data transformations, outlier analysis, covariates, assumption corrections, and multiplicity controls (i.e., Type I error controls).

Preregistration is designed to solve the problems presented in this scenario, if a researcher adequately details the connections between the components of their study. What actually happens in pre-registration? As a classroom exercise, the lead author required students to find articles that included a Preregistered Open Science Badge (Kidwell et al., 2016). Badges were designed to increase the uptake of open science practices, namely to preregister studies, share data, and share materials, and these badges appear to have positive outcomes (Rowhani-Farid, Allen, \& Barnett, 2017). While 60+ journals use the Open Science Badges, SAGE and specifically, the Association for Psychological Science (APS) journals appear to be one of the only journals that clearly signal these badges (i.e., they are displayed next to the article link online, on the article page, and on the PDF; the Psi Chi Journal also uses this approach). Other journals only show the badges on the formal PDF of the article, and it is unclear from many of the journals if they simply do not have articles with open information (Franco, Malhotra, \& Simonovits, 2014) or simply do not display badges in any way in their articles. Given the lack of visible signalling of the badges, the class focused on APS journals. In general, the students found the preregistrations to match the studies as presented in their journal articles with minor deviations and inconsistencies (Buchanan et al., 2018).

However, it is unclear if these minor deviations and inconsistencies occurred simply because of the journals selected or because articles with poor matches from preregistration to final paper were rejected by the journal editors/reviewers. Additionally, undergraduates may not be able to judge with sufficient nuance the differences between plans and actuality. Claesen, 
Gomes, Tuerlinckx, and Vanpaemel (2019) examined preregistrations found in Psychological Science from 2015 to 2017 . All studies showed at least one deviation from the pre-registered plan, which may be expected because of unexpected challenges in the research study (Nosek et al., 2018) or the editorial process (Bloomfield, Rennekamp, \& Steenhoven, 2018). Critically, Claesen et al. (2019) found that all but one study did not disclose this difference between plan and paper, and approximately $41 \%$ did not reach a level of detail to accurately judge the plan or the pre-registered plan was inaccessible (i.e., not clear which plan was correct, no time stamp). Therefore, even with preregistration, we find that the logical mapping between hypotheses, methods, data, and statistical analysis may not be consistent or clear. Szollosi et al. (2019) further argue that preregistration does not necessitate a well-defined relationship between theory, measurement, and methodology, and requiring preregistration may not improve theoretical reasoning. Aside from these inconsistencies in preregistration literature, there are also other concerns from scientists. Goldin-Meadow (2016) argued that preregistration can prevent new discoveries, suggesting that data-driven approaches can foster new findings. However, these concerns have been countered with the success of registered reports, which are preregistered papers that are accepted in principal before the study has been completed (Chambers, 2015; Chambers, Feredoes, Muthukumaraswamy, \& Etchells, 2014). Despite the conversation about preregistration, Moore (2016) suggests that preregistration will increase transparency in psychological science, which may lead to increased trust in published findings.

The concerns about the limitations of preregistration are legitimate, and we suggest, instead, focusing on the planning of research in any way possible. While many suggest that preregistration discourages exploratory research (Goldin-Meadow, 2016), we believe that even exploratory projects should have a plan; it would still be a questionable research practice to simply collect as much data as possible and then test it for every conceivable relationship. 
Further, this behavior likely encourages other questionable research practices, for instance only reporting the significant findings and variables after exploring the data (Simmons et al., 2011). A solidified approach to preregistration is necessary. Therefore, we make the following suggestions:

1) Create a roadmap of the relationship between your supporting literature and hypotheses. For each hypothesis, detail the measurement of the relevant variables in your study. In this stage, it is important to understand questionable research practices in measurement (Flake \& Fried, 2019) in order to accurately measure the construct of interest. The type of data collection (i.e., between subjects, repeated measures) and measurements will define potential appropriate statistical analyses.

a) At this stage, other important considerations, such as power and sample sizes, should be reviewed as part of the measurement plan. For all studies, focus on constructing a plan to accurately measure your parameters, rather than to power a statistical test (Kelley, Darku, \& Chattopadhyay, 2018; Kelley \& Rausch, 2011). This approach is particularly useful for descriptive and exploratory studies but can be applied to nearly all studies.

b) In order to fully understand the connection between your measurement and analysis, consider creating simulated data and pre-run your analysis. This approach can solidify the output format of the data (e.g., what are the columns and what do they represent), the potential transformations of the data (i.e., summed or total scores), and how to proceed with the analysis. Generating even a few example participant data points can be a useful 
exercise for planning a study, as key missed measurement issues may be highlighted at this stage. Another approach would be to create an expected data dictionary, or document that outlines the information and attributes provided in a dataset (Buchanan et al., 2019). Most software programs/survey collection platforms allow for the generation of dummy data to assist in this suggestion.

2) Use built in human subjects' requirements as an opportunity to plan your study, rather than a chore to be completed. Institutional Review Board requirements vary widely by location but often minimally require details about the methods and variables involved in a study. Similar to a preregistration, these can provide time-stamped hypotheses and analysis plans.

3) Consider expanding the suggested roadmap into a registered report (Chambers, 2013; Nosek \& Lakens, 2014). As noted, registered reports are study plans that are submitted for acceptance to a journal before the study has been carried out, and over 200 journals have adopted this type of report (see https://cos.io/rr/). Registered reports allow for critical review of your proposed roadmap, with the ability to improve measurement, theory, or statistical plans before the work has been completed. By receiving reviews before data collection or analysis on secondary data, one may fix critiques prior to study completion, rather than trying to appease reviewers after the fact.

Does it matter if you preregister your study? It seems unlikely that we may reach a stage of journals only accepting preregistered studies. The current publications on preregistration indicate a strong focus on the prevention of questionable research practices and hypothesizing after results are known in reaction to the "replication crisis" that has gripped the social sciences. 
What does matter is the need for a clear blueprint between the supporting literature, hypotheses, and study plan that may alleviate the need to find "something publishable" from a dataset. Preregistration provides templates to complete a plan, along with registered reports for publication acceptance before starting a study. Scientists can grow to have a deeper understanding of their project by thoughtfully identifying how to map theories to testable hypotheses and translate these into collectable data and meaningful analyses in a realistic way. Together, new blueprints that elucidate connections between different study components can help bring researchers together and potentially further reproducible and meaningful science. 


\section{References}

Bloomfield, R. J., Rennekamp, K. M., \& Steenhoven, B. A. (2018). No system is perfect: Understanding how registration-based editorial processes affect reproducibility and investment in research quality. SSRN Electronic Journal.

https://doi.org/10.2139/ssrn.3118687

Buchanan, E. M., Crain, S. E., Cunningham, A. L., Johnson, H. R., Stash, H. E., Papadatou-Pastou, M., ... Aczel, B. (2019). Getting started creating data dictionaries: How to create a shareable dataset. https://doi.org/10.31219/osf.io/vd4y3

Buchanan, E. M., Marshall, C. Z., Compton, O. L., Sibert, C. E., Stash, H., Kelley, H., ... Chadwell, E. (2018, October 29). Week 11 - Pre-registration. Retrieved December 24, 2019, from Open Science Framework website: https://osf.io/tzq4x/

Chambers, C. D. (2013). Registered reports: a new publishing initiative at Cortex. Cortex; a Journal Devoted to the Study of the Nervous System and Behavior, 49(3), 609-610. https://doi.org/10.1016/j.cortex.2012.12.016

Chambers, C. D. (2015). Cortex's registered reports. Cortex; a Journal Devoted to the Study of the Nervous System and Behavior. Retrieved from https://www.elsevier.com/editors-update/story/peer-review/cortexs-registered-reports

Chambers, C. D., Feredoes, E., Muthukumaraswamy, S. D., \& Etchells, P. J. (2014). Instead of "playing the game" it is time to change the rules: Registered Reports at AIMS Neuroscience and beyond. AIMS Neuroscience, Vol. 1, pp. 4-17.

https://doi.org/10.3934/neuroscience.2014.1.4

Claesen, A., Sara, Gomes, Tuerlinckx, F., \& Vanpaemel, W. (2019). Preregistration: Comparing dream to reality. https://doi.org/10.31234/osf.io/d8wex

Flake, J. K., \& Fried, E. I. (2019). Measurement schmeasurement: Questionable measurement 
practices and how to avoid them. https://doi.org/10.31234/osf.io/hs7wm

Franco, A., Malhotra, N., \& Simonovits, G. (2014). Publication bias in the social sciences: Unlocking the file drawer. Science, 345(6203), 1502-1505. https://doi.org/10.1126/science.1255484

Gelman, A., \& Loken, E. (2013). The garden of forking paths: Why multiple comparisons can be a problem, even when there is no "fishing expedition" or "p-hacking" and the research hypothesis was posited ahead of time. Retrieved from http://www.stat.columbia.edu/ gelman/research/unpublished/p_hacking.pdf

Goldin-Meadow. (2016). Why preregistration makes me nervous. APS Observer. Retrieved from https://www.psychologicalscience.org/observer/why-preregistration-makes-me-nervous

Gonzalez, J. E., \& Cunningham, C. A. (2015). The promise of pre-registration in psychological research. Psychological Science Agenda. Retrieved from https://www.apa.org/science/about/psa/2015/08/pre-registration

Kelley, K., Darku, F. B., \& Chattopadhyay, B. (2018). Accuracy in parameter estimation for a general class of effect sizes: A sequential approach. Psychological Methods, 23(2), 226-243. https://doi.org/10.1037/met0000127

Kelley, K., \& Rausch, J. R. (2011). Sample size planning for longitudinal models: accuracy in parameter estimation for polynomial change parameters. Psychological Methods, 16(4), 391-405. https://doi.org/10.1037/a0023352

Kerr, N. L. (1998). HARKing: Hypothesizing after the results are known. Personality and Social Psychology Review, 2(3), 196-217. https://doi.org/10.1207/s15327957pspr0203_4

Kidwell, M. C., Lazarević, L. B., Baranski, E., Hardwicke, T. E., Piechowski, S., Falkenberg, L.-S., ... Nosek, B. A. (2016). Badges to acknowledge open practices: A simple, low-cost, effective method for increasing transparency. PLoS Biology, 14(5), e1002456. 
https://doi.org/10.1371/journal.pbio.1002456

Leis-Newman, E. (2011). Securing tenure: On the tenure track? Here are four keys to making sure you get the ultimate prize. PsycEXTRA Dataset.

https://doi.org/10.1037/e545582011-035

Moore, D. A. (2016). Preregister if you want to. The American Psychologist, 71(3), 238-239. https://doi.org/10.1037/a0040195

Nelson, L. D., Simmons, J., \& Simonsohn, U. (2018). Psychology's renaissance. Annual Review of Psychology, Vol. 69, pp. 511-534.

https://doi.org/10.1146/annurev-psych-122216-011836

Nosek, B. A., Ebersole, C. R., DeHaven, A. C., \& Mellor, D. T. (2018). The preregistration revolution. Proceedings of the National Academy of Sciences of the United States of America, 115(11), 2600-2606. https://doi.org/10.1073/pnas.1708274114

Nosek, B. A., \& Lakens, D. (2014). Registered Reports: A method to increase the credibility of published reports. Social Psychology, 45(3), 137-141.

https://doi.org/10.1027/1864-9335/a000192

Nosek, B. A., \& Lindsay, D. S. (2018). Preregistration becoming the norm in psychological science. APS Observer. Retrieved from https://www.psychologicalscience.org/observer/preregistration-becoming-the-norm-in-psych ological-science

Rowhani-Farid, A., Allen, M., \& Barnett, A. G. (2017). What incentives increase data sharing in health and medical research? A systematic review. Research Integrity and Peer Review, 2, 4. https://doi.org/10.1186/s41073-017-0028-9

Simmons, J. P., Nelson, L. D., \& Simonsohn, U. (2011). False-positive psychology: Undisclosed flexibility in data collection and analysis allows presenting anything as significant. 
Psychological Science, 22(11), 1359-1366. https://doi.org/10.1177/0956797611417632

Szollosi, A., Kellen, D., Navarro, D., Shiffrin, R., van Rooij, I., Van Zandt, T., \& Donkin, C. (2019). Is preregistration worthwhile? https://doi.org/10.31234/osf.io/x36pz

Van den Akker, O., Weston, S. J., Campbell, L., Chopik, W. J., Damian, R. I., Davis-Kean, P., ... Bakker, M. (2019). Preregistration of secondary data analysis: A template and tutorial. https://doi.org/10.31234/osf.io/hvfmr

van 't Veer, A. E., \& Giner-Sorolla, R. (2016). Pre-registration in social psychology-A discussion and suggested template. Journal of Experimental Social Psychology, 67, 2-12. https://doi.org/10.1016/j.jesp.2016.03.004

Wagenmakers, E.-J., Wetzels, R., Borsboom, D., van der Maas, H. L. J., \& Kievit, R. A. (2012). An agenda for purely confirmatory research. Perspectives on Psychological Science: A Journal of the Association for Psychological Science, 7(6), 632-638.

https://doi.org/10.1177/1745691612463078 